\title{
Response
}

\section{Projection of Diabetes Prevalence in Korean Adults for the Year 2030 Using Risk Factors Identified from National Data (Diabetes Metab J 2019;43:90-6)}

\author{
Inkyung Baik \\ Department of Foods and Nutrition, College of Science and Technology, Kookmin University, Seoul, Korea
}

I would like to express my sincere thanks for Dr. Koo's comments on the recently published article about the projected prevalence of diabetes mellitus (DM) in Korean adults [1]. In this article, the current and future prevalence estimates of DM were calculated among adults aged 30 years or older because this age-range was used in the report of the Korea National Health and Nutrition Examination Survey [2]. Accordingly, it is reasonable that the projected prevalence for the year 2030 presented in the article [1] was relatively higher than an estimate among adults aged 20 to 79 years proposed by the International Diabetes Federation (IDF) [3]. In addition, the method which was used to obtain the IDF's estimates is likely to produce underestimated projections as indicated in an earlier report [4]. If the denominator is changed into the number of adults aged 20 to 79 years, the prevalence of DM at the year 2030 would be $18.9 \%$ ( $13.8 \%$ in women and $23.9 \%$ in men) using the same method of the article [1]. Furthermore, if the denominator is the number of the total Korean population, the prevalence of DM at the year 2030 would be $14.2 \%$ (10.0\% in women and $18.3 \%$ in men), which is comparable to the prevalence (15.3\%) projected in the total United States population [5]. The IDF has also speculated that the projections of DM prevalence for the United States and Republic of Korea at the year 2030 would be similar [3].

Being intrigued by Dr. Koo's suggestion regarding agegroup-specific projections, further analysis has been attempt- ed; projected prevalence estimates are 10.6\% (7.8\% in women and $13.4 \%$ in men) for adults aged 20 to 64 years (the younger) and $43.6 \%$ (31.7\% in women and $55.4 \%$ in men) for those aged 65 to 79 years (the older) when considering the population projection at 2030 [6]. Before considering it, however, 9.1\% (6.3\% in women and $11.9 \%$ in men) in the younger and $26.8 \%$ (23.1\% in women and $30.4 \%$ in men) in the older have been estimated. Interpreting these results, I agree with Dr. Koo's speculation regarding an increasing trend in DM prevalence among male adults due to increasing prevalence of risk factors including obesity as well as among male and female elders due to an increasing size of the elderly population. Thus, the suggestion of forecasting age-specific prevalence of DM is quite worthy of being considered.

\section{CONFLICTS OF INTEREST}

No potential conflict of interest relevant to this article was reported.

\section{REFERENCES}

1. Baik I. Projection of diabetes prevalence in Korean adults for the year 2030 using risk factors identified from national data. Diabetes Metab J 2019;43:90-6.

2. Ministry of Health and Welfare, Korea Centers for Disease Con-
Corresponding author: Inkyung Baik (D) https://orcid.org/0000-0002-9524-4344 Department of Foods and Nutrition, College of Science and Technology, Kookmin University, 77 Jeongnung-ro, Seongbuk-gu, Seoul 02707, Korea

E-mail: ibaik@kookmin.ac.kr
This is an Open Access article distributed under the terms of the Creative Commons Attribution Non-Commercial License (http://creativecommons.org/licenses/by-nc/4.0/) which permits unrestricted non-commercial use, distribution, and reproduction in any medium, provided the original work is properly cited. 
trol and Prevention. Korea health statistics 2015: Korea National Health and Nutrition Examination Survey (KNHANES VI-3). Sejong: Korea Centers for Disease Control and Prevention; 2016.

3. Whiting DR, Guariguata L, Weil C, Shaw J. IDF diabetes atlas: global estimates of the prevalence of diabetes for 2011 and 2030. Diabetes Res Clin Pract 2011;94:311-21.

4. Guariguata L, Whiting D, Weil C, Unwin N. The International
Diabetes Federation diabetes atlas methodology for estimating global and national prevalence of diabetes in adults. Diabetes Res Clin Pract 2011;94:322-32.

5. Rowley WR, Bezold C, Arikan Y, Byrne E, Krohe S. Diabetes 2030: insights from yesterday, today, and future trends. Popul Health Manag 2017;20:6-12.

6. Statistics Korea. Population projections for Korea: 2010-2060. Daejeon: Statistics Korea; 2011. 